\title{
CONSTRUÇÃO DE UM PARADIGMA DE CUIDADO DE ENFERMAGEM PAUTADO NAS NECESSIDADES HUMANAS E DE SAÚDE
}

\author{
The building of a paradigm for nursing care lined in the human and health needs \\ Construcción de un paradigma de cuidado en enfermería pautado en las necesidades \\ humanas y de salud
}

Denize Cristina de Oliveira ${ }^{1}$

Antonio Marcos Tosoli Gomes²

Ana Paula Munhen de Pontes ${ }^{3}$

Camila Perroni Marouço da Costa ${ }^{4}$

\section{RESUMO}

Propõe-se a problematização da adoção dos conceitos de necessidades básicas humanas e de necessidades de saúde para a constituição das bases epistemológicas de um conceito de cuidado de enfermagem que possibilite a orientação da ação profissional para o seu atendimento, no contexto da pós-modernidade. A proposição básica desenvolvida é a do acesso às necessidades humanas e de saúde através das representações sociais, com suas derivações conceituais para o cuidado em enfermagem e suas tecnologias de trabalho. Demonstra-se que as necessidades de saúde podem ser proveitosamente acessadas através da exploração tanto das dimensões objetivas, quanto das subjetivas do processo saúde-doença, como representadas pelos sujeitos e subjacentes às suas demandas. Discute-se, ainda, a necessidade de repensar o modelo tecnológico de trabalho e a de estabelecer novas bases teóricas para o processo de cuidar em enfermagem, a partir das necessidades negociadas entre os sujeitos individuais e coletivos e os profissionais nas instituições de saúde.

Palavras-chave: Determinação de necessidades de cuidados de saúde. Cuidados de enfermagem. Assistência à saúde.

\begin{abstract}
This article discusses the concepts of basic human and health needs in order to set up the epistemological grounds for a concept of nursing care which allows the guidance for the professional action during the treatment, in the post modern context. The basic formulation addresses the access to human and healh needs through social representations, along with their conceptual derivations for nursing care and their work technologies. Evidence is provided that health needs can be productively addressed through the exploration of their objective and subjetive dimensions in the health-disease process, as represented by their subjects and underlying demands. Further discussion is offered on the need to alter the technological work model and the need to set up new theoretical frameworks for the nursing care process, from the requirements negotiated between the individual and collective subjects and professionals in health.
\end{abstract}

Keywords: Needs assessment. Nursing care. Delivery of Health Care .

\section{Resumen}

Se propone la problematización de la adopción de los conceptos de necesidades básicas humanas y de necesidades de salud para la constitución de las bases epistemológicas de un concepto de cuidado en enfermería que posibilite la orientación de la acción profesional para su atención en el contexto posmoderno. La propuesta básica desarrollada es la del acceso a las necesidades humanas y de salud a través de las representaciones sociales, con sus derivaciones conceptuales para el cuidado en enfermería y sus tecnologías de trabajo. Se demuestra que las necesidades de salud pueden ser provechosamente alcanzadas a través del estudio tanto de las dimensiones objetivas como de las subjetivas del proceso salud-enfermedad, conforme representadas por los sujetos y subyacentes a sus demandas. Se discute también la necesidad de repensar el modelo tecnológico de trabajo y de establecer nuevas bases teóricas para el proceso de cuidado en enfermería, a partir de las necesidades negociadas entre los sujetos individuales y colectivos y los profesionales en las instituciones de salud.

Palabras clave: Evaluación de necesidades. Atención de Enfermería. Prestación de Atención de Salud

\footnotetext{
${ }^{1}$ Doutora em Saúde Pública e Pós-doutora em Psicologia Social; Professora Titular do Depto. de Fundamentos de Enfermagem e do Programa de Pós-graduação em Enfermagem, Faculdade de Enfermagem, Universidade do Estado do Rio de Janeiro. Líder do Grupo de Pesquisa Promoção da Saúde e Práticas de Cuidado de Enfermagem e Saúde de Grupos Populacionais. Rio de Janeiro - R. Brasil. E-mail: dcouerj@gmail.com; ${ }^{2 D o u t o r ~ e m ~ E n f e r m a g e m ; ~ P r o f e s s o r ~ A d j u n t o ~ d o ~ D e p t o . ~ d e ~ E n f e r m a g e m ~ M e ́ d i c o-~}$ Cirúrgica e do Programa de Pós-graduação em Enfermagem, Faculdade de Enfermagem, Universidade do Estado do Rio de Janeiro. Pesquisador do Grupo de Pesquisa Promoção da Saúde e Práticas de Cuidado de Enfermagem e Saúde de Grupos Populacionais. Rio de Janeiro - RJ. Brasil. E-mail: mtosoli@gmail.com; ${ }^{3}$ Mestre em Enfermagem; Professora Substituta do Depto. de Enfermagem Médico-Cirúrgica, Faculdade de Enfermagem, Universidade do Estado do Rio de Janeiro. Pesquisadora do Grupo de Pesquisa Promoção da Saúde e Práticas de Cuidado de Enfermagem e Saúde de Grupos Populacionais. Rio de Janeiro - RJ. Brasil. E-mail: anamunhen@gmail.com; ${ }^{4} G$ raduada em Enfermagem; Mestranda do Programa de Pós-graduação em Enfermagem, Faculdade de Enfermagem, Universidade do Estado do Rio de Janeiro. Aluna do Grupo de Pesquisa Promoção da Saúde e Práticas de Cuidado de Enfermagem e Saúde de Grupos Populacionais. Rio de Janeiro - RJ. Brasil. E-mail: camila.perroni@hotmail.com
} 


\section{INTRODUÇ̃̃O}

Tomado como campo de produção intelectual, ${ }^{1}$ o campo de saber da enfermagem vem se estruturando em função de um permanente refletir sobre as suas práticas e sobre a construção do seu objeto de atenção. Nesse sentido, concebida como prática social, a enfermagem tem procurado definir a especificidade do seu objeto e do seu processo de trabalho, em consonância com os outros processos de trabalho do campo da saúde.

A proposição do presente texto é a de refletir sobre o conceito de necessidades humanas e de saúde e sua pertinência ao cuidado de enfermagem/de saúde, a partir da análise de publicações. Parte-se da premissa de que os conceitos de necessidades humanas e de saúde se encontram subjacentes à definição de cuidado de enfermagem, da mesma forma que permanece subjacente às práticas de saúde em geral.

Considerando o cuidado de enfermagem como tecnologia de trabalho, adota-se a proposição segundo a qual o trabalho em saúde se organiza, de acordo com cada período histórico, em torno de modelos de ação, com objetos de trabalho específicos, determinando instrumentos, produtos e resultados. ${ }^{2}$ Esses modelos partem de uma certa definição de necessidades, que podem ser rotuladas como necessárias, sociais ou radicais. Por outro lado, cada modelo de atenção tem sua objetivação em um determinado processo de trabalho, que determina o espaço e a concepção de necessidades que o subsidiará. ${ }^{2}$

No que se refere a essa questão, as necessidades vêm sendo concebidas como importantes indicadores de avaliação da qualidade das tecnologias de trabalho de saúde. Isso vem configurando uma nova equação, que anula a diferenciação entre necessidades e demandas, tão apregoadas no conhecimento clássico da saúde, como sinônimos de verdade (a primeira) e de ficção (a segunda). Desta forma, a efetividade dessas tecnologias passa a ter como um dos seus elementos de avaliação o atendimento das necessidades, que é entendido como a satisfação dos clientes com a atenção profissional prestada.

A satisfação dos clientes, portanto, é tomada como medida de avaliação da atenção em saúde e em enfermagem, com referência a conceitos como o de aceitabilidade, que é a adaptação do cuidado aos desejos, expectativas e valores dos pacientes e de suas famílias. Em grande parte, a aceitabilidade depende das valorizações subjetivas dos clientes com relação ao desempenho dos profissionais e dos serviços. A rigor, não apenas o indivíduo passa a ser considerado nessa equação, mas também a comunidade, visto que a sua legitimidade é conferida pela aceitabilidade do cuidado para a comunidade ou a sociedade em geral. Dessa forma, a equação da equidade assume contornos mais complexos, pelos quais o que os indivíduos elegem como satisfatório deve ser levado em consideração juntamente com o que a sociedade julga satisfatório.

Nessa proposição, a medida da satisfação das necessidades é dada pela atitude do sujeito para com o sistema de atenção à saúde adotado e com o qual tenham tido contato. A qualidade do cuidado é julgada pela sua conformidade com um grupo de expectativas ou padrões que derivam de três fontes: (1) a ciência do cuidado à saúde; (2) os valores e expectativas individuais; (3) os valores e expectativas dos grupos sociais (suas representações). Em consequência, a qualidade não pode ser julgada apenas em termos técnicos por profissionais de saúde; as preferências de pacientes e da sociedade como um todo também devem ser levadas em conta.

Partindo dessas premissas conceituais propõe-se a problematização da adoção dos conceitos de necessidades básicas e de necessidades de saúde para a constituição das bases epistemológicas de um conceito de cuidado de enfermagem que possibilite a orientação da ação profissional para seu atendimento.

\section{PILARES DE UM PARADIGMA ASSISTENCIAL PARA O CUIDADO DE ENFERMAGEM}

A proposição básica que se pretende desenvolver neste texto é a do acesso às necessidades humanas e de saúde através das representações sociais, permitindo derivações conceituais para o cuidado em enfermagem importantes para a sua efetivação enquanto tecnologia de trabalho.

\section{As Necessidades Básicas e de Saúde e suas Contribuições para a Conceitualização do Cuidado de Enfermagem}

No marco da pós-modernidade, deve-se reconhecer que o conhecimento passa por uma crise de paradigmas, que se expressa em todas as áreas do conhecimento, particularmente naqueles que têm no humano o seu objeto de trabalho. Na enfermagem, essa crise tem se expressado no questionamento das categorias de conhecimento tradicionalmente fundamentais do campo. Essas categorias são identificadas por diferentes autores como "necessidades", "resultados", "cliente", "pessoa-ambiente", "terapêutica de enfermagem" ; como "campo de energia" e "campo humanístico";; e como "intervenção", "submissão", "conservação", "apoio" e "ampliação" , revelando perspectivas diversas nas teorias que embasam a enfermagem.

Uma segunda forma de expressão dessa conflitualidade paradigmática refere-se ao questionamento do conceito de cuidado de saúde e de enfermagem, que acaba por exigir a problematização do que se concebe como necessidades, sejam elas formalizadas como "demandas", "necessidades de cuidado", "necessidades de serviços", "direitos", ou outras. Apesar de não explicitadas, diferentes representações subjacentes de necessidades estão presentes tanto nas teorias 
de enfermagem, quanto nos modelos teóricos de cuidado propostos, o que revela a importância desse conceito para o saber/fazer de enfermagem.

A delimitação conceitual das necessidades humanas básicas ressurgiu suscitando considerável interesse analíticocrítico por parte dos setores intelectuais e políticos. Contudo, a literatura acadêmica sobre essa temática ainda padece de imprecisões e ambiguidades. Muitas vezes, a expressão "necessidades humanas básicas" tem uma conotação tão ampla, relativa e genérica, que dificulta a identificação dos conteúdos, contornos e particularidades desse conceito, confundindo-o com uma dimensão restrita ao biológico e ao seu caráter instrumental.

Por outro lado, deve-se considerar a existência de importantes correntes de pensamento que rejeitam a proposição de que existam, de fato, necessidades básicas comuns a todos, que sejam objetivamente identificáveis e cuja satisfação poderia ser planejada e gerida de forma sistemática e bem-sucedida, seja em nível individual ou coletivo. Esta rejeição sustenta um certo ceticismo sobre a possibilidade de se ter, do ponto de vista teórico, um corpo de conhecimentos coerente e objetivo sobre essa temáticas.

Não obstante a diversidade dessas abordagens, é possível observar que elas têm se encaminhado para uma polarização que aglutina, majoritariamente de um lado, as que identificam necessidades básicas com estados subjetivos e relativos de carecimentos e, minoritariamente de outro, as que tomam essas necessidades como um fenômeno objetivo, passível de generalização, apoiado na existência de necessidades universais, conforme a seguir:

\section{...o procedimento dialógico, discursivo, realizado no mundo da vida, ou seja, na sociedade, é condição fundamental para que se tracem normas universais, simétricas e recíprocas de justiça e moralidade, contribuindo para a definição de necessidades humanas que são universais. $6: 85$}

A posição dominante é aquela sustentada pelos teóricos que, apoiando-se nas evidências sobre diferenças interpessoais e culturais, têm privilegiado o subjetivismo e 0 relativismo no trato das necessidades humanas básicas, dentro de um entendimento econômico-capitalista das necessidades, pautada na lógica de mercado. Esses autores partem da premissa de que, se não há necessidades comuns que sejam vivenciadas coletivamente e que sirvam de parâmetro para a formulação de ações e para a implementação de políticas públicas, não haverá melhor mecanismo para satisfazê-las do que o mercado. Desta forma, é o mercado que tem interesse e predisposição para maximizar demandas individuais, ampliando o leque de aspirações particulares, para manter-se dinâmico, promissor e lucrativo. ${ }^{7}$ Essa é a lógica que tem amparado a expansão da atenção privada à saúde que vem se desenvolvendo no Brasil, através dos planos e seguros de saúde.

Com base nessa tendência têm proliferado interpretações de necessidades humanas básicas que sistematicamente as confundem com outras noções, tornandoas inespecíficas e determinando formas de satisfação confusas e voluntaristas. Nesse sentido, do ponto de vista conceitual, necessidades humanas e sociais são frequentemente consideradas como: falta ou privação de algo objetivo ou subjetivo; preferência por determinado bem ou serviço em relação a outro; desejo de quem, psicologicamente, se sente carente de alguma coisa; compulsão por determinado tipo de consumo, movida pela dependência ou pelo uso repetitivo ou viciado desse consumo; demanda como procura por satisfação econômica, social ou psicológica de alguma carência. ${ }^{8}$

Há, ainda, quem confunda necessidade com motivação, expectativa ou esperança de obter algo de que se julga merecedor, por direito ou promessa, conforme observado na teoria de Maslow, com base na qual Wanda Horta constituiu a sua teoria das necessidades humanas básicas.

Das várias concepções inespecíficas de necessidades, algumas se tornaram mais conhecidas em função da sua recorrência, principalmente aquelas centradas nos aspectos somáticos e psicológicos dos indivíduos em suas demandas relativas. Na base dessas concepções, observa-se uma forte justificação de origem ética, que expressa a convicção de que é moralmente mais consistente equiparar "necessidades" a "preferências subjetivas", porque só os indivíduos ou grupos particulares sabem das suas demandas e, por isso, são mais capazes do que as instituições de traçar os objetivos e prioridades que melhor Ihes convêm. Essa concepção tem contribuído para 0 ataque às políticas de bem-estar social e o conseqüente desmantelamento de direitos sociais conquistados, fortalecendo uma visão individualista e apolítica das necessidades humanas básicas.

Buscando dar visibilidade às diferentes formas de apropriação das concepções de necessidades humanas pelas proposições teóricas da enfermagem, foi observada a existência de quatro padrões. Esses variam segundo o papel dos fatores sociais na teoria, que atribui determinada amplitude ao conceito de necessidades; 0 caráter universal ou grupal das necessidades; a hierarquização das necessidades ou a sua concepção como elemento isolado; a superposição das necessidades à noção de sintomas; o grau de interdependência assumido entre os cuidados de enfermagem e as necessidades. ${ }^{9}$

Os padrões de incorporação destacados foram os seguintes: 1) A ação de enfermagem fundamentada nas necessidades humanas básicas, tendo sua grande expressão nas proposições de Horta, ${ }^{10}$ mas também presente nas proposições de Orem, Abdellah, Leininger, Orlando e Wiedenbach;2) As necessidades como resposta a estímulos de adaptação humana, especialmente considerado nos trabalhos de Nightingale, ${ }^{11}$ mas também presente nas proposições de Roy, Neuman e Rogers; 3) As necessidades humanas como 
construções subjetivas e resultantes de processos de significação, observadas nos trabalhos de Peplau, Sisca, Newman, Parse, Patterson e Zderad, Travelbee e King; 4) As necessidades humanas como expressão ética e moral do homem que se apresenta especialmente nas obras de Leopardi, ${ }^{12}$ mas também na de Leininger.

\section{As Representações Sociais como Tradutoras de Necessidades Humanas e de Saúde e as Tecnologias de Cuidado}

A compreensão da noção de cuidado de enfermagem implica considerar o conceito de processo de trabalho e o de tecnologia de trabalho com o qual se articula. Nessa proposição

... a tecnologia dos processos de trabalho é algo que se constitui dentro dos mesmos, apontando ao mesmo tempo para suas dimensões técnicas e sociais. O seu estudo implica no saber e seus desdobramentos em técnicas materiais e não materiais que, ao darem um sentido técnico ao processo, também Ihe atribuem um sentido social articulado (...) refere-se ainda aos nexos técnicos estabelecidos no interior do processo de trabalho entre a atividade operante e os objetos de trabalho. ${ }^{13: 66}$

As tecnologias de trabalho, dentre elas as tecnologias de cuidado de enfermagem, vistas a partir das representações sociais, possibilitam um novo olhar, que não mais as considera como esfera normativa, estritamente técnica e cientificamente determinada, mas enquanto expressão das subjetividades, que se constituem em um dado espaço social.

Isso implica em conceber o duplo caráter assumido pelo cuidado de enfermagem: o primeiro, enquanto ação legitimada pelo - e legitimadora do - conhecimento científico; o segundo enquanto construção da realidade e, nessa medida, como função da experiência pessoal, da ação criativa e do conhecimento de base empírica.

Nessa perspectiva, o cuidado de enfermagem e as demais práticas profissionais de saúde ganham sentido e contexto como ações intencionais, institucionalmente localizadas e socialmente determinadas. Enquanto forma particular de agir no mundo, a tecnologia do cuidado de enfermagem é constituída por um saber e por seus desdobramentos materiais e não materiais, inseridos na produção de serviços de saúde.

Definido como tecnologia de trabalho, o cuidado de enfermagem se insere no processo de trabalho em saúde, que está baseado no princípio da existência de "algo antes" e "algo depois". Uma vez que o trabalho se consubstancia em produtos e resultados, este deve ser captado como instrumento teórico, que permita a identificação de elementos e dimensões através dos quais se pode compreender a dinâmica das práticas de grupos profissionais específicos, como os enfermeiros, e da organização de uma forma mais geral.

Cabe destacar que, nessa perspectiva, os indivíduos envolvidos no processo de cuidado, ou seja, profissionais de enfermagem e clientes, são vistos como atores que constroem e negociam continuamente arranjos sociais, através de sua vivência e de suas relações; dentro dessa negociação, encontram-se as necessidades diferentemente concebidas por uns e por outros. ${ }^{2}$

Em pesquisa desenvolvida sobre a tradução das necessidades de saúde entre usuários de serviços de saúde foi observado que

...as necessidades apontadas pelos usuários dos serviços revestem-se de uma característica mais instrumental, qual seja, a de dar respostas a demandas que se colocam no dia a dia dos sujeitos, mas sem se divorciar de princípios que essa população vai agregando às suas representações de saúde e de doença. ${ }^{9: 195}$

Por outro lado, os profissionais de enfermagem na atualidade definem tais necessidades tanto a partir das possibilidades colocadas pelo conhecimento reificado, quanto pelo processo de trabalho em saúde, muito mais do que pelas demandas dos usuários dos serviços onde atuam.

No estudo anteriormente referido o que se observou quanto às necessidades de saúde concebidas pelos profissionais foi

uma compatibilização socioconceitual entre as 'demandas vivas' do cliente e as necessidades tradicionalmente reconhecidas pelo saber de enfermagem. O momento vivido e seus significados foram expressos pelas representações sociais do processo saúde doença dos entrevistados, expressando as suas próprias necessidades e expectativas. $^{9: 207}$

Consequentemente, ao focalizar o processo de trabalho e as tecnologias de cuidado, é possível perceber as dimensões formais e informais presentes nessa determinação das necessidades e verificar como e sob quais facetas essas concepções se manifestam, sejam elas mais técnicas, políticas, sociais ou culturais.

Dentre os elementos técnicos que determinam as concepções de necessidades pelos profissionais, são consideradas, por exemplo, as normas prescritivas teóricas e tecnológicas, supostamente organizadas e orientadas para a consecução de objetivos, para a obtenção de resultados. Os elementos socioculturais e psicossociais expressam-se, na perspectiva da tecnologia administrativa formal, como ruídos que embaraçam a racionalidade técnica. Os elementos participantes das relações interpessoais e da subjetividade 
individual, tais como conflitos, disputas, irracionalidades, intuições, capacidade de negociação e criatividade, conformam as dimensões afetiva e ideológica, que se mostram, a um só tempo, competitivas, antagônicas e complementares nesse processo.

Ao buscar definir bases para uma concepção de cuidado de enfermagem nesta análise, procura-se dar ênfase ao agente, ao ator, ao sujeito ético, ${ }^{2}$ no caso o profissional de enfermagem, que convive com uma determinada racionalidade, em geral não emancipatória. Porém, é nesse ambiente que o profissional age, sendo capaz ou não de transformar o processo de trabalho e suas tecnologias, dentre elas a do cuidado de enfermagem, e as finalidades e necessidades que o determinam. Para alcançar essa mobilidade de ação é preciso buscar vias que possibilitem o trânsito de uma dimensão técnico-teórica das necessidades para uma outra dimensão de valores e significados, que considere as demandas da população como expressões legítimas das suas necessidades, e estas enquanto inscrições nas suas representações do processo saúde-doença. ${ }^{9}$

A existência de uma dimensão ética é imposta pela natureza do cuidado de enfermagem, uma vez que a geração e o consumo do produto desse cuidado se dão, simultaneamente, na interação que se estabelece entre o profissional e o cliente. Daí decorre outra problemática a ser considerada: a efetividade das tecnologias depende da pactuação, por clientes e profissionais, de uma interação produtiva. Mas essa interação produtiva implica na aceitação de uma negociação de saberes sobre as necessidades e os cuidados possíveis para o seu atendimento, porque, em última instância, é essa negociação que deverá definir quais ações profissionais são reconhecidas como cuidado e o grau de satisfação da clientela com elas, bem como o sucesso das ações de cuidado como ações terapêuticas.

Ao discutir as categorias definidoras do processo saúdedoença, observa-se que

... as representações de saúde e doença não são
constituídas essencialmente na polaridade do corpo
são e do corpo doente, mas avançam na sua
compreensão como parte do sistema produtivo da
sociedade, indicada na categoria 'atividade'; revelam
ainda a valorização da qualidade de vida, na
categoria 'estado sociocultural'; e lhe atribuem um
sentido de força interior, objetiva e subjetiva,
apontada nas categorias 'estado espiritual'e 'atitude
no cotidiano.'. 9:198

Nesse sentido, coloca-se a necessidade de pensar o cuidado de enfermagem como uma tecnologia de trabalho com contornos específicos, não ancorada no saber clínico-biomédico, que na interação com os saberes dos outros campos científicos e profissionais, e também com os saberes construídos pelos sujeitos cuidados, vai se transformando, moldando e se reconstruindo, tendo por base as representações sociais constituídas nos diferentes grupos em interação. Nessa trajetória, tende a abandonar uma concepção idealista e tecnicista do cuidado, que o associa a impedimentos técnicos e não inseridos em processos sociais e organizacionais específicos, demonstrando que o objeto de trabalho da enfermeira se confunde com o próprio processo de trabalho. ${ }^{13}$ No entanto, a posição observada da enfermeira no processo de trabalho em saúde caracteriza-se pela inserção em uma hierarquia que se ancora no grau de "gravidade" da patologia ou "do caso" e é centrada na atenção clínica e individual. Observa-se, assim, que o objeto da ação de cuidado dessa profissional é geralmente associado a "desvios da normalidade" do sujeito individual, dado que o sujeito coletivo é mencionado apenas enquanto objeto de práticas idealizadas, conforme observado em estudo sobre as práticas dos enfermeiros no Programa de Saúde da Criança. ${ }^{14}$

No entanto, no plano do saber, encontram-se proposições orientadas pela consideração do objeto de trabalho da enfermagem sobre outras bases, segundo as quais o trabalho da enfermeira deveria apontar para uma direção diversa da do trabalho médico, ou seja, para o sujeito coletivo e para os saberes de base humanista.

Embora a noção, bem como os conteúdos relativos aos direitos humanos sejam históricos, estes devem, ainda, ser configurados como balizadores das necessidades a serem consideradas pelos enfermeiros, através de ações e relações individuais e coletivas, construídas entre profissionais-clientelacontexto institucional.

Tomar as necessidades humanas básicas na sua dimensão de autonomia significa compreender que elas só podem ser definidas na relação com os sujeitos que as geram. Não obstante, é imprescindível conceber um limiar humano, portanto social, para as necessidades consideradas no seu polo coletivo, na medida em que uma resposta social geradora de preocupação ativa e genuína em relação aos problemas de saúde só pode se constituir a partir da negociação de interesses e vontades profissionais e dos clientes. A relação estabelecida entre ações de cuidado de enfermagem e a satisfação de necessidades exige a crítica concreta ao modo como estas são traduzidas, apresentadas e incorporadas no trabalho em saúde e, especificamente, de enfermagem. Importa superar a sua definição a partir de critérios técnicos e históricos, segundo os quais as demandas são tidas como distintas das necessidades, concebidas as primeiras como falsas e as segundas como verdadeiras.

0 alvo da intervenção em enfermagem precisa ser deslocado do corpo em desequilibrio, conforme concebido pela maior parte das construções teóricas do cuidado de enfermagem, para os sujeitos em suas expressões coletivas e individuais, a partir das suas condições concretas - objetivas e subjetivas - de existência. Para isso, é necessário avançar de uma valorização da dimensão biológica humana para uma valorização da dimensão psicossocial, sem alienar o indivíduo/ 
sujeito do seu contexto de vida, pois, se tomadas essas dimensões como fatores isolados no indivíduo, com a finalidade de ordenar a ação cuidadora, mantém-se uma situação de primazia do conhecimento técnico sobre o conhecimento do senso comum, assim como a relação de desigualdade ora existente entre eles.

As necessidades em saúde, de expressão coletiva, não podem ser isoladas e individualizadas, já que elas apenas assumem sentido no corpo social onde são geradas. As tecnologias de cuidado de enfermagem se destinam a abranger o espaço individualizado e o coletivo, a partir das determinações do processo saúde-doença, mas não apenas delas. Nesse processo está também implicada a subjetividade coletiva constituída, considerando que as necessidades de saúde extrapolam categorias identificáveis em nível individual. Isso não significa negar a importância da manifestação e satisfação das necessidades individuais, particulares, ou mesmo das ações individualizadas de cuidado. Ao contrário, significa valorizar a ação cuidadora a partir de uma perspectiva em que esses elementos se integrem ao fazer junto a sujeitos sociais concretos.

De fato, uma visão da totalidade e da historicidade humana requer o questionamento do modo como se identificam as necessidades, do modo como elas são explicadas e das intervenções assumidas para a sua satisfação. A identificação e a análise das diferentes necessidades dos sujeitos ou segmentos sociais, nos espaços coletivos e individualizados, devem orientar as decisões acerca de quais ações e processos devem ser encaminhados para o seu enfrentamento, bem como para a definição das tecnologias de trabalho mais adequadas a esse objetivo. Dessa forma, os instrumentos de trabalho devem permitir a identificação social das necessidades de saúde.

Defendemos a utilização das representações sociais ${ }^{15 ; 16}$ para a identificação das necessidades de saúde. Essas representações, acessadas a partir dos profissionais e dos sujeitos coletivos da atenção à saúde, permitem a tradução das necessidades de saúde, em uma relação de confronto produtivo, gerador de uma ação pactuada entre profissionais e população.

Assim, considerando o referencial orientador deste texto, qual seja, a definição de necessidades humanas como direitos, precisa-se levar em conta a construção da autonomia dos sujeitos individuais e coletivos, tomando-se ela própria, a autonomia, como necessidade humana fundamental.

Nesse sentido, vale salientar a importância de se avançar no desafio de construção de uma concepção de cuidado de enfermagem que leve em conta a multidimensionalidade humana, relevando as suas implicações para as práticas profissionais e institucionais. Importa conhecer como o enfermeiro vem enfrentando as transformações que se colocam no cotidiano da prática de cuidar, à luz das tecnologias cotidianamente incorporadas, e de que forma isso implica nas suas atitudes e modos de agir. 17:172

A construção de uma nova subjetividade profissional implica, portanto, rever as concepções de necessidades e também de tecnologias de trabalho e de cuidado, incluindo nestas a dimensão subjetiva característica do humano que é o profissional e também dessa dimensão na clientela por ele atendida.

Dessa forma, à reinterpretação das necessidades e à mudança na processualidade da sua definição seguir-se-á o desafio da construção das tecnologias e processos de cuidado de enfermagem adequados, ou seja, da especificação de quais ferramentas, quais processos, quais saberes, qual intersubjetividade são mais adequados à construção dessa nova racionalidade.

Uma nova qualidade assistencial que considere a integralidade humana, o processo de vida e seus riscos inerentes, bem como o respeito incondicional à autonomia dos sujeitos, depende do adequado estabelecimento das necessidades e das tecnologias de trabalho em saúde. Nesse processo, o trabalho e o trabalhador da enfermagem devem contribuir para a expressão e satisfação dessas necessidades, segundo um modo novo e articulado de reconhecê-las, descrevêlas e atendê-las ou transformá-las.

\section{CONCLUSÕES}

A partir do exposto considera-se que um reposicionamento profissional deve se dar enquanto busca de novos caminhos e estratégias para a assunção, pela enfermeira, de um paradigma de cuidado coerente com as necessidades sentidas e latentes dos indivíduos e, assim, enquanto compromisso com a melhoria da qualidade de vida e de saúde da população.

Este texto poderá contribuir para a prática profissional de enfermeiros e outros profissionais de saúde na medida em que o reposicionamento profissional referido e as necessidades representadas sejam adotados como ponto de partida para as ações de cuidado de saúde e de enfermagem, qualificando esse cuidado e aproximando-o do universo psicossocial dos grupos atendidos.

As dificuldades particulares de cada área profissional, bem como das áreas de saber que representam, suas raízes históricas, seus paradigmas epistemológicos, não são elementos imutáveis; eles se transformam, tal qual as representações, a partir das práticas que efetivam. Considera-se, portanto, mais produtivo pensar o saber em enfermagem e as práticas que esse saber sustenta, dentro dessa dialética que comporta a mudança.

Para que as práticas de cuidado de enfermagem não se reduzam a uma concepção clínico-biológica da saúde, mesmo tendo por fundamento concepções mais ampliadas, as diversas áreas de saber implicadas nesse cuidado precisam ser incorporadas dentro de uma perspectiva de complementaridade, sem obscurecer a relação necessidades - cuidado, própria da enfermagem. Nessa medida, se é possível questionar a capacidade e suficiência do saber clínico em estabelecer 


\section{Cuidado de Enfermagem e Necessidades Humanas e de Saúde}

necessidades de saúde, deve-se questionar também porque a ele se prender no estabelecimento de saberes, ações e tecnologias de cuidado aceitáveis para o enfrentamento dos problemas e necessidades de saúde. Já não seria o momento de questionar a circunstância (porque não se trata de mais do que isso) de apenas um saber informar a compreensão dos problemas de saúde, bem como as concepções de necessidades humanas/de saúde?

A questão essencial na análise aqui desenvolvida são as rupturas necessárias para a transformação do processo de trabalho em enfermagem, e da sua principal tecnologia - 0 processo de cuidar - implicando na superação e transformação de conceitos, hábitos, procedimentos, enfim, de representações e práticas. Implica, também, a ruptura com as dicotomias preventivo-curativo e individual-coletivo, assim como a busca de um compromisso com a qualidade das ações de cuidado que respondam às necessidades e demandas da população usuária dos serviços de saúde.

Conclui-se a necessidade de repensar o modelo tecnológico de trabalho e a de estabelecer novas bases teóricas e conceituais para o processo de cuidar em enfermagem, a partir das necessidades negociadas entre os sujeitos individuais e coletivos e os profissionais nas instituições de saúde, marcando a especificidade desse cuidado e o que o diferencia do padrão genérico de cuidar, tão apregoado nas publicações no momento atual.

\section{REFERÊNCIAS}

1. Bourdieu P. 0 poder simbólico. Rio de Janeiro: Difel/Bertrand; 1989.

2. Gonçalves RBM. Práticas de saúde: processos de trabalho e necessidades. São Paulo : CEFOR; 1992.

3. Meleis A. Theoretical nursing: development and progress. Philadelphia: JB Lippincott Company; 1985.

4. Marriner-Tomey A. Modelos y teorias en enfermeria. Philadelphia: Harcourt Brace; 1994.

5. Fawcett J. Analysis and evaluation of conceptual models of nursing. Philadelphia: F.A. Davis Company; 1995.

6. Habermas J. Consciência moral e agir comunicativo. Rio de Janeiro: Tempo Brasileiro; 1983-1989.

7. Santos BS. Pela mão de Alice: o social e o político na pós-modernidade. Porto: Ed Afrontamento; 1994.

8. Pereira PAP. Necessidades humanas: subsídios à crítica dos mínimos sociais. São Paulo: Cortez;2000.
9. Oliveira DC. A enfermagem e as necessidades humanas básicas: 0 saber/fazer a partir das representações sociais [tese professor titular]. Rio de Janeiro: Faculdade de Enfermagem, Universidade do Estado do Rio de Janeiro/UERJ; 2001.

10. Horta WA. Processo de enfermagem. São Paulo: EPU; 1979.

11. Nightingale F. Notas sobre enfermagem. São Paulo: Cortez; 1989.

12. Leopardi MT. Teorias em enfermagem: instrumentos para a prática. Florianópolis: Ed. Papa Livros; 1999.

13. Gonçalves RBM. Tecnologia e organização das práticas de saúde: características tecnológicas do processo de trabalho na rede estadual de centros de saúde de São Paulo [tese]. São Paulo: Faculdade de Medicina, Universidade de São Paulo; 1986.

14. Oliveira DC. A promoção da saúde da criança: análise das práticas cotidianas através do estudos de representações sociais [tese]. São Paulo: Faculdade de Saúde Pública, Universidade de São Paulo; 1996.

15. Herzlich C. Santé et maladie: analyse d'une représentation sociale. Paris: Mouton; 1969.

16. Jodelet D. La representación social: fenómenos, concepto y teoría. In: Moscovici S, organizador. Pensamiento y vida social: psicologia social y problemas sociales. Buenos Aires: Paidos; 1986. p. 469-93.

17. Silva RC, Ferreira MA. A tecnologia em saúde: uma perspectiva psicossociológica aplicada ao cuidado de enfermagem. Esc Anna Nery. 2009 jan/mar; 13 (1): 169-73. 